\title{
The Developing Approaches to Evolutionary Epistemology: An Historical Perspective
}

\author{
Ren Qiaohua, \\ School of Marxism, Shenyang Jianzhu University \\ Shenyang, China, 110168 \\ tony805@163.com
}

\author{
Zhang Jingzhu \\ School of Marxism, Shenyang Jianzhu University \\ Shenyang, China, 110168 \\ 1058954011@qq.com
}

\begin{abstract}
Evolutionary epistemology has been developed in various historical stages that responds to different approaches, namely, adaptationism, non-adaptationism and interactionism. This paper gives an account of three approaches in terms of the hot debate on confirming the mechanism of biological evolution and elucidates why these approaches have been developed in different contexts. It is suggested that the development of evolutionary epistemology should be of implications on the studies of epistemology.
\end{abstract}

Keywords-evolutionary epistemology, adaptationism, non-adaptationism, organism, environment

\section{INTRODUCTION}

Nathalie Gontier, an American professor of philosophy at the University of Lisbon, has addressed the different approaches to evolutionary epistemology. She suggests that the traditional approach is based upon biological adaptionism whereas this approach has ignored the mental process of organism. By contrast, the non-adaptionism approach is proposed to compensate for the flaws of the traditional ones. [1] Her understandings of evolutionary epistemology has been drawn increasingly attention around the field of bio-philosophy. From the available literature, more focuses have been on exploring the general evolutionary epistemology mechanism but little is known about the theoretical basis for the changing approaches to evolutionary epistemology. Hereby, it is of significance for us to clarify the reasons for these changes and to account for implications for traditional epistemology.

\section{THE ADAPTATIONISM APPROACHES TO EVOLUTIONARY EPISTEMOLOGY: NATURAL SELECTION BASED}

Since the early 20th century, adaptationism prevailed in biology has directly influenced the approaches to evolutionary epistemology. By the approach to adaptationism, organism and environment are viewed as two separate entities, which interact only in a selective process, but are independent of each other during its development [2]. Neo-Darwinism as a dominant theory in that period strictly insists on the dualism and maintains that organisms are passively selected by environment. In the early period of evolutionary epistemology, it follows the ways by giving priority to natural selection when interpreting biological evolution, including cognitive development.

\section{A. Natural selection - as an absolute power}

The biologist, Symons (1990) sees the trait as a result of

This paper is sponsored by Projects of the National Social Science Foundation of China (15CZX014), "EEM Program's Theoretical Development and Methodological implications” adaptation, believing that the organism gene in the gene pool corresponds to the trait. From parents to future generations, genes are adapted to this channel. Genes can influence human cognitive constructs.[3] Since genes will affect human cognitive constructs, behavioral ecologists gradually stand in the adaptationist position to test the variation and successful reproduction fitness from the view of natural induced traits and the variation of experimental traits. In the eyes of Buss (1995), this approach poses two problems: (1) The evolutionary adaptedness of organism coincides with evolutionary adaptedness of environment whereas the previously adapted environment is different from the existing environment. (2) The psychological mechanism common to mankind is unique.[4]

Dusenbery (1996) tries to explicate the origin of species' specificity from three points: (1) Biological adaptation conforms to the psychological mechanism. (2) biological variation drives natural selection. (3) The genetic mechanism is transmitted.[5] As far as he is concerned, biological adaptation is the way of determining the psychological mechanism. Through natural selection, human cognitive ability is the product of evolution, so the interpretation of human traits must be consistent with the natural selection theory. Human behavior science and other studies are also required to follow the approach to natural selection. As it should be, the explanation of other animal traits also follows this approach. In addition, from a view of species specificity, even if biological variation drives natural selection, different species in the face of evolutionary adaptive environment will have different problems with the corresponding solutions, so that biological adaptation itself has become the key to solve the adaptive problems. It is true that, from the biogenetic gene transfer mechanism, all the complex mechanisms of biology are evolved from thousands of genes. Recombination of genetic factors between individuals is a prerequisite for maintaining complex mechanisms. Recombination of natural selection and sexual reproduction often leads to a relatively uniformity of complex adaptive design.

Adaptationism approaches to Evolutionary epistemology just follow Dusenbery's interpretation of the priority to natural selection, but is it possible that this adaptationism approach can be carried out in different contexts? 


\section{B. The external environment- as a dominant power}

Wuketits (1989) states briefly that the adaptationist approaches to evolutionary epistemology involve two basic assumptions: (1) The sense organ of any organism will be adapted to the external world. (2) What the organism perceives is a real but simplistic mirror or part of the external world.[6] Wuketits's assumptions point to the adaptability of cognitive structures and the external world's structure. The adaptationism approach to evolutionary epistemology attempts to examine the adaptability of biological cognitive phenotypic traits, by use of the term "function" to refer to the traits by satisfying special needs, emphasizing the implications of environmental selection. Hereby, adaptation is also defined from the perspective of the evolution of species. On this basis, Godfrey-Smith(2001) distinguishes with three paradigms of adaptionism :[7]

(1) Empirical adaptationism. Maynard Smith is seen as a typical representative in this area. He indicates that natural selection has a strong, ubiquitous causal power whereas the biological variation can be ignored. From a view of natural selection, the results of the evolutionary process can be predicted and explained. Natural selection promotes the formation of organism structures, including cognitive structures. There are no other evolutionary factors that can produce such a powerful force.

(2) Explanatory adaptationism. The neurologist Daniel Dennett is treated as a typical representative in this area. He highlights natural selection has a unique explanatory power and theory of selection can solve the "design" problems of organism as well as problems of the interactions between environment and organism. In other words, natural selection is seen as a naturalistic and non-theological solution to the "design" problem, as the only the process to result in a complex physiological and psychological mechanisms.

(3) Methodological adaptationism. Ernst Mayrt is regarded as a typical representative in this area. He encourages scientists to solve biological system problems by examining the adaptive function and design. In order to elucidate the biological function, an important prerequisite of evolutionary epistemology will include the understanding and description of cognitive mechanisms, so as to solve the special adaptive problems. Methodological adaptationism treats biocompatibility as an organizational principle, with special ways of studying organisms.

In addition to the above three adaptationism, Murphy(2003) also proposes forward-looking adaptationism conception. [8] Forward-looking adaptationism begins with the assumptions of the Environment of Evolutionary Adaptedness (EEA) and the problems faced by biological ancestors in the environment. So its strategy is to assume that the designing traits can effectively solve the adaptive problem. In other words, from an empirical point of view, they support special adaptation and indirectly support the EEA hypothesis. It is suggested that EEA is seen as an important concept of adaptationism approach to evolutionary epistemology. In examining non-human species, the old problems of species are often studied as existing problems, so they assume that non-human species can adapt to the living environment, as the
EEA has not been changed. In contrast, during the process of human evolution, the adaptation to earlier environment is different from adaptation to current environment, so it seems that the EEA of human has been changed in this respect.

The early study of evolutionary epistemology is based upon the theory of evolutionary biology. The standpoint of adaptionism has been defensed by theory of selection. Due to the dominant position of adaptionism in biology during the first half of the 20th century, many scholars tend to argue that this approach is legitimate to reveal the problems of mental organization with special psychological structures. This approach to evolutionary epistemology must start with the discovery and description of biological adaptation, because the complex functional organization of the organism is embedded into the organ design by means of the adaptive mechanism. Natural selection is known as the only designer of biological functional organ. Thereby, the success of evolutionary biology lies so much in the use of adaptionism approaches to explain the functional design of organic traits.

This approach to evolutionary epistemology is encouraged to regard the physiological trait of biological cognition as a successful extension of the other mental traits of the organism, such as vertebrate eyes. Under the influence of this approach, evolutionary epistemologists advocate that the interpretation of the mind needs to start from the relationship between mind and body, so the cognitive structure and function is no longer a by-product of other traits. Thus, early evolutionary epistemology supports the principle of adaptionism, particularly in favor of the theoretical basis of adaptive maximization to biological behavior. However, this approach has long ignored the important process of knowledge generation, namely, mental construction.

\section{THE NON-ADAPTATIONISM APPROACH TO EVOLUTIONARY EPISTEMOLOGY: INITIATIVES BASED}

Adaptationism follows the principle that natural selection is viewed as most important evolutionary casual powers. Non-adaptionists advocate that the evolutionary mechanism cannot be confined to the adaptation to the external environment, but also should include historical contingencies and other factors. Thus, the refutation of adaptationism is finally focused on the discussion of whether random factors can play an important role in the process of biological evolution. In the study of evolutionary epistemology, adaptationists hold that the biological evolutionary mechanism of cognition is based upon an external natural selection, whereas non-adaptationists do believe that cognitive biological evolution is determined by both the external environment and organism itself, rather than their complete passively adaptation to the environment.

\section{A. Subjective initiative of the organism}

After the 1970s, biological system theory has been constructed by indicating that we can't ignore the internal selection of organism in a process of cognition. This theory reflects the inherent initiative of organism. In terms of Darwinism, biological system theory does not deny the externally environment selection, but attaches importance to the internal selection. Since internal selection is directed at the 
organism's structure that will constrain their own adaptation and evolution. The biological adaptation is defined by organism itself, rather than that by environment or mysticism. In terms of evolutionary epistemology, biological system theory transcends the scope of natural selection theory, which overcomes the one-sidedness of adaptationism, providing the communicative platform between cognitive science and biology.

\section{B. Adaptive Subjectivity in a complex biological system}

The non-adaptionism approach to evolutionary epistemology lays stress on the fact that biological adaptation is not defined by environment, but by organism itself. In particular, although the biological system and environment share a history together, but are not subject to independent evolution.

Firstly, the organism is taken as the hardcore to describe and explain their role in the process of evolution. Gould (1983) states briefly that the organism's appearance and embryonic development form are inherited, which brings constraint to its future changes and adaptation. In many cases, the evolutionary path reflects the genetic pattern rather than the existing environmental requirements.[9] $\mathrm{He}$ departs from the evolutionary process of biological genetic mechanism, describing the complexity of biological systems.

Secondly, the organism is a complex, orderly system that involves the interactive elements. From organs and tissues, to cells and molecules, it is considered that the organism is a highly ordered system with different hierarchical structures that allow organs and functions to correspond to each other. The basic structure of the chromosome can achieve the goals of collective movement and transformation coherently, highlighting the general high degree of coordination between time and space. The ultrastructure of living cells is a complex network structure, on the grounds of certain rules to produce the overall pulse and transformation, and to maintain the unity of the system.

Thirdly, the organism itself is a complex and active system that can run, fly, swim, dig and nest, etc., which demonstrate the complexity of the organism system, so that the organism is not only affected by environment, but also affect the environment. This indicates that organism has hierarchical structures of the developmental system, which achieve the goals of self-maintenance through the components' exchanges in system. It also explains why the organism is not entirely determined by environment.

\section{THE NEW APPROACHES TO EVOLUTIONARY EPISTEMOLOGY: INTERACTIONISM BASED}

Munz (2001) has suggested that this new approach to evolutionary epistemology indicates that knowledge comes from the interaction between organism and environment.[10] The empiricist argues that rationality is based on the inductive process of experience, through the associative process to draw reasoning conclusions. The rationalist believes that knowledge is born of innate elements and rational knowledge is seen as the logical relationship between ideas. In knowledge sociology, knowledge is a result of agreement between different cognitive subjects.
Since the $21^{\text {st }}$ century, the focuses of evolutionary epistemology have been on the constructivist approach, in which coherent theory and constructivism theory play a central role. In accordance with this new approach, knowledge derives from the relationship between organism and environment, so that the tension between adaptionism and non-adaptionism has been maintained through addressing the interaction between organism and environment

\section{A. Transforming correspondence theory to coherent theory}

The traditional approach to evolutionary epistemology points to the correspondence between cognition and the external world, believing in "one to one" mapping relations between environment and organism. This stereotypical correspondence theory often overlooks the fact that the organism is a active system and that knowledge comes from the interaction between environment and organism. The biological system itself is integrating the internal mechanism with the external environment through the feedback loop, coupled with the special nature of the evolution of human cognitive mechanism, so that this new approach to evolutionary epistemology strongly initiates the integration of internal and external mechanisms.

The coherent theory reflects the internal initiative of organism. This approach to evolutionary epistemology vigorously advocates the perceived coherence is reduced to the functional ones. The external information can be accurately perceived by organism in an given environment, which can be coherently transformed into internal structure or the life systematic organization. This forms a feedback chain from the organism to the outside world. Each organism's interpretation of reality, to a certain extent, is not simply described by their sense organs. It is seen as transcendence to epistemology of analyst philosophy. In particular, it is important to note that in terms of coherent theory, the specific organization of organism determines their perceived differences in the external world, which confirms the basic relationship between the organism and environment. It is not free from the environment but is integrated into the environment. In this sense, environment can influence the organism that can adapt to it. The organism also acts on the environment, resulting in environmental behavior. It is of great survival significance for the creatures in the interaction between environment and organism.

\section{B. A constructivism approach}

Wuketits(2000) formulated three hypotheses in accordance with this new approach: (1) Cognitive is an active biological system function;(2) Knowledge comes from the complex interaction between organism and environment; (3) Cognition is a continuous process of trial and error .[11] This new approach is in line with the constructivism theory.

The constructivism theory reflects the initiative of organism's internal selection. Lewontin (2000) does not see the organism as a negative element of selection, whereas he introduces an evolutionary constructivist approach that distinguishes between different aspects of organism and environment: The evolutionary constructivist approach focuses not only on the interrelationships between the organism and the environment, but on the internal processes of organism and environment. The organism is determined by itself. The 
elements from the external environment include their own environment or niche, and to a large extent determine how they relate to each other. The organism not only selects its environment, but also constructs its own niche. Organisms continue to actively change their environment and learn to provide the external conditions of the environment. [12]

This new approach to evolutionary epistemology is not to examine how organism evolves by adapting to a given environment, but rather a way of thinking about how organism and environment interact, confirming that organism do not passively adapt to the external world but actively build and transform the external environment, so that it can flourish in a changing environment. In other words, life is a process of information processing, whose development depends on the interaction between the given environment and species. Therefore, for the sake of survival, the organism actively interacts in different ways by pursuing knowledge included in the organism's structure.

\section{CONCLUDING REMARKS}

Evolutionary epistemology is seen as a multidisciplinary field, which includes biology, neurophysiology, cognitive science, psychology, philosophy, etc. Evolutionary epistemology continually faces challenges from biological facts due to the constantly changing research paradigms within disciplines. This makes evolutionary epistemology necessary to be changed for improvement. With the development of modern scientific methods, the problems of biodiversity and the complexity of evolution have to be faced by epistemology. The philological intuitive or speculative approach cannot directly be used to understand thoroughly about organism's behavior in a microscopic sense, let alone knowing clearly about their perception to environment. Consequently, the overall development of epistemology must be accompanied by the overall development of biology, neurophysiology and other disciplines. From the implications of adaptionionism to epistemology, it is considered that cognition is a kind of passive adaptation or a mechanical reactive behavior. From the implications of non-adaptionionism to epistemology, it is believed that active cognitive behavior is seen as a general feature of all species.

\section{ACKNOWLEDGEMENT}

This paper is sponsored by Projects of the National Social Science Foundation of China (15CZX014), "EEM Program's Theoretical Development and Methodological implications”.

\section{REFERENCES}

[1] Gontier, Nathalie. "Evolutionary epistemology as a scientific method: a new look upon the units and levels of evolution debate." Theory in Biosciences129.2-3 (2010): 167-182.

[2] Lewontin, Richard C. The triple helix: Gene, organism, and environment Harvard University Press, 2001. APA

[3] Symons, Donald. "Adaptiveness and adaptation." Ethology and Sociobiology11.4-5 (1990): 427-444.

[4] Buss, David M. "Evolutionary psychology: A new paradigm for psychological science." Psychological inquiry 6.1 (1995): 1-30.

[5] Dusenbery, D.B. Life at a small scale: The behaviour of microbes. New York: Scientific American Library, 1996: 68.

[6] Wuketits, F.M. “Cognition: A non-adaptationist view.” La Nuova Critica, 1989, 9 (1): 5

[7] Godfrey-Smith, P. Adaptationism and Optimality. Oxford: Oxford University Press, 2001: 335-357.

[8] Murphy D. Adaptationism and and Psychological Explanation. Boston, Ma: Kluwer Academic Publishers, 2003.

[9] Gould, S.J. Hen's Teeth and Horse's Toes: Further Reflections in Natural History. Harmondsworth: Penguin Books, 1983: 156.

[10] Munz, Peter. Philosophical Darwinism: on the origin of knowledge by means of natural selection. London: Routledge, 2001: 9.

[11]Wuketits, F.M. Functional Models of Cognition: Self-organizing Dynamics and Semantic Structures in Cognitive Systems Dordrecht: Kluwer Academic Publishers, 2000b: 27.

[12] Lewontin, Richard. The Triple Helix: Gene, Organisms and Environment. Cambridge: Harvard University Press. 2000: 51-64. 